\title{
Multiparametric MRI analysis of morphologico-functional features of DCIS - correlation with the grade of nuclear atypia
}

\author{
V. LEHOTSKA*, K. RAUOVA, L. VANOVCANOVA \\ $2^{\text {nd }}$ Radiology Department, Faculty of Medicine, Comenius University in Bratislava, Bratislava, Slovakia \\ *Correspondence: viera.lehotska@ousa.sk
}

Received May 9, 2017/ Accepted August 5, 2017

\begin{abstract}
The aim was to analyze morphologico-functional characteristics of ductal carcinoma in situ (DCIS) in breast MRI to assess signs correlating with the degree of nuclear atypia. From 636 malignant lesions, 114 were DCIS (17.92\%): 44 cases of high-grade (38.60\%), 37 intermediate (32.45\%), 33 low-grade (28.95\%). MRI characteristics - T2 signal intensity (SI), shape, margins, contrast enhancement, peripheral/ductal enhancement, kinetic curve, presence of restriction of diffusion were correlated with the nuclear grade of DCIS. Statistical analysis was performed, statistical significance and Odds ratio (OR) were calculated. Signs of high-grade lesions were low SI in T2w ( $\mathrm{p}=0.042)$, nonhomogeneous contrast enhancement $(\mathrm{p}=0.012)$, wash-out phenomenon $(\mathrm{p}=0.04)$, high SI in diffusion weighted imaging (DWI) $(\mathrm{p}<0.0001)$, restriction of diffusion in apparent diffusion coefficient map $(\mathrm{p}<0.0001)$. DWI and wash-out phenomenon reached the highest OR (56.00, 9.76). Breast MRI using DWI and multiparametric analysis provides important information about the degree of nuclear atypia.
\end{abstract}

Key words: breast carcinoma, ductal carcinoma in situ, multiparametric magnetic resonance imaging, diffusion weighted imaging, tumor grading

Ductal carcinoma in situ (DCIS) is a non-invasive form of malignant breast lesion that is characterized by proliferation of malignantly transformed intraductal cells of the terminal ductolobular unit. There is no invasion through the basal membrane and no infiltration of surrounding structures, but it is considered a high risk lesion and a precursor of invasive carcinoma [1]. In 30-50\% DCIS transforms gradually to invasive ductal carcinoma, and approximately in $4 \%$ of cases there are micrometastases in the axillary lymph nodes at the time of diagnosis [2].

Its potential to progress to invasive carcinoma increases with the degree of nuclear atypia. High-grade DCIS is typically fast-growing lesion with high mitotic activity and in a short period of time of intraductal spreading it may progress to a high-grade invasive ductal carcinoma [3]. Low-grade lesions persist and grow for a relatively long time inside the ductal tree, after its invasions through the basal membrane a low-grade invasive carcinoma develops [1]. This indicates that recognition of the grade of nuclear atypia plays a key role in the further management of DCIS.

In the last decade magnetic resonance imaging (MRI) has played the most important role in DCIS diagnostics even though its benefit in preoperative management of DCIS is still discussed [4]. The pathophysiological mechanism of the post contrast enhancement of this process remains still unclear. There is an evident difference between the morphology and post contrast behavior of a healthy duct and a duct affected by DCIS [5]. It is caused by increased permeability of basal membrane of the duct containing DCIS which allows the gadolinium chelate contrast agent to enter into the impaired duct [5]. This process is distinguishable in MR examination as a pathological ductal enhancement [6]. Character of post contrast enhancement of DCIS can be regarded as a biomarker of the biological behavior of the lesion. The sensitivity of MRI in detection of DCIS correlates with its degree of nuclear atypia, the sensitivity of MRI in DCIS diagnostics grows with the probability of its transformation into invasive form [4]. It is not rare that low-grade lesions remain without any enhancement while high-grade lesions usually present themselves by pathological ductal pattern of enhancement $[7,8]$. Furthermore, there are other MRI characteristics of DCIS that might provide helpful information about its degree of nuclear atypia: signal intensity in $\mathrm{T} 2 \mathrm{w}$, diffusionweighted imaging (DWI) and apparent diffusion coefficient (ADC) map, shape and margins of the lesion, as well as its post contrast behavior-pattern of enhancement and kinetic 
curves. The combination of morphological, functional and molecular information offered by multiparametric MRI promises to make it a successful tool for breast cancer diagnosis [9]. The aim of this study was to analyze all MRI features, correlate them with the degree of nuclear atypia and to select the most significant signs that would be relevant in differentiation of grade of nuclear atypia of DCIS.

\section{Patients and methods}

In the period 10/2013-09/2015, 636 malignant breast lesions were diagnosed and histologically proven, out of which 114 (17.92\%) were confirmed as pure DCIS. Lesions detectable in ultrasound (Philips iU22, Belgium, 2012) were verified by an ultrasound guided core cut biopsy (with withdrawal of minimally 5-6 samples). Those with mammographic pathologic microcalcifications (Hologic, LORAD, Dimensions, USA, 2010) underwent vacuum assisted biopsy (Hologic Multicare Platinum, USA 2012 and ATEC Suros, Hologic, USA, 2012) with multiple samples removal.
All lesions were confirmed by histopathological analysis as DCIS. None of these had any signs of invasion or microinvasion. According to the degree of nuclear atypia (nuclear grade), the whole group was divided into three subgroups: high-grade DCIS group with 44 cases $(38.60 \%)$, intermediate grade DCIS with 37 cases $(32.45 \%)$ and low-grade group with 33 cases (28.95\%).

According to our currently used internal recommendations for DCIS management, all patients with diagnosed ductal carcinoma in situ underwent breast MRI examination. All MR studies were conducted at 3T Magnetom Verio (Siemens, Belgium, 2010). Patients were positioned prone into the dedicated 16-channel breast coil. The MRI protocol included T2 fat sat axial images, DWI using b values of 0,50 , 850, T2 Tirm cor, T1 axial and dynamic contrast enhancement (DCE) axial. In the dynamic study, the contrast agent gadoteridol (ProHance, Bracco Diagnostics, Singen, Germany) was administered at a dosage $0.1 \mathrm{mmol} / \mathrm{kg}$. The first non-enhanced series were followed by seven contrast enhanced series, each with acquisition time of $60 \mathrm{~s}$.

Table 1. Frequency of morphological and functional MRI characteristics for high, intermediate a low-grade DCIS.

\begin{tabular}{|c|c|c|c|c|}
\hline $\begin{array}{ll}\text { MR signs } & \text { Degree of nuclear atypia } \\
\end{array}$ & $\begin{array}{l}\text { High-grade } \\
\mathrm{n}=44\end{array}$ & $\begin{array}{l}\text { Intermediate } \\
\quad \mathbf{n}=\mathbf{3 7}\end{array}$ & $\begin{array}{c}\text { Low-grade } \\
\mathbf{n}=33\end{array}$ & Total \\
\hline \multicolumn{5}{|l|}{ T2 signal } \\
\hline Low & 11 & 7 & 2 & 20 \\
\hline Intermediate & 27 & 17 & 14 & 58 \\
\hline High & 6 & 13 & 17 & 36 \\
\hline \multicolumn{5}{|l|}{ Shape and margins } \\
\hline Regular & 0 & 0 & 0 & 0 \\
\hline Lobular & 0 & 0 & 0 & 0 \\
\hline Irregular & 39 & 37 & 33 & 109 \\
\hline Spiculated & 5 & 0 & 0 & 5 \\
\hline \multicolumn{5}{|l|}{ Post contrast enhancement } \\
\hline Homogeneous & 2 & 7 & 9 & 18 \\
\hline Non-homogeneous & 42 & 30 & 24 & 96 \\
\hline \multicolumn{5}{|l|}{ Ring enhancement } \\
\hline yes & 0 & 0 & 0 & 0 \\
\hline no & 44 & 37 & 33 & 114 \\
\hline \multicolumn{5}{|l|}{ Ductal enhancement } \\
\hline yes & 24 & 21 & 18 & 63 \\
\hline no & 20 & 16 & 15 & 51 \\
\hline \multicolumn{5}{|l|}{ Kinetic curves } \\
\hline TIC 1 (continual SI increase) & 8 & 9 & 21 & 38 \\
\hline TIC 2 (plateau) & 19 & 21 & 10 & 50 \\
\hline TIC 3 (“wash-out" phenomenon) & 17 & 7 & 2 & 26 \\
\hline \multicolumn{5}{|l|}{ DWI Signal } \\
\hline Low & 2 & 10 & 24 & 36 \\
\hline High & 42 & 27 & 9 & 78 \\
\hline \multicolumn{5}{|l|}{ ADC Value } \\
\hline Decrease & 40 & 25 & 11 & 76 \\
\hline Increase & 4 & 12 & 22 & 38 \\
\hline ADC coefficient $\times 10^{-3} \mathrm{~mm}^{2} / \mathrm{s} \pm \mathrm{SD}$ & $0.87 \pm 0.12$ & $0.95 \pm 0.10$ & $1.09 \pm 0.06$ & $0.96 \pm 0.19$ \\
\hline
\end{tabular}


All breast MRIs were analyzed by two skilled breast radiologists (16 and 10-year experiences). In the morphological group of signs, we focused on the shape and margins of lesions, signal intensity in $\mathrm{T} 2 \mathrm{w}$, character of post contrast enhancement (homogeneity and presence of ring or ductal enhancement). According to the pharmacokinetics of extracellular contrasts agent, kinetic curves were calculated for each lesion. Signal intensity in DWI and presence of restrictions of diffusion in ADC map completed the functional characteristics of analyzed lesions. The most important and reliable MR signs and parameters correlating with the degree of nuclear atypia were identified (Table 1).

For selected MR characteristics, logistic regression analysis was performed with regards to the nuclear grade of the lesions, followed by calculation of statistical significance (p) and Odds ratio. For statistical analysis we used software Acastat version 7.0. The level of statistical significance for particular signs was calculated by Chi-Square test (StatCalc, version 7.1.2, Excel 2010 ) and was set as $p<0.05$. Logistic regress analysis was performed by software MedCalc ${ }^{\oplus}$, version 12.4.0, 2003-2013.

\section{Results}

In our retrospective multiparametric study, all DCIS behaved as lesions with heterogeneous character. Signal intensity in T2w differed significantly between groups of high and low-grade DCIS. Low T2 signal of high-grade lesions (11 out of $44,25 \%, p=0.0422$ ) and high signal intensity of low-grade (17 out of $33,51.52 \%, p=0.0007$ ) were found to be relevant for nuclear grade evaluation with $\mathrm{p}$ being statistically significant (Table 2, Figure 1A, Figure 3A). This difference of T2 signal intensity was not so obvious between groups of high and intermediate DCIS $(\mathrm{p}=0.1267)$ (Figure $2 \mathrm{~A})$, though the Odds ratio reaching 3.62 made low signal intensity in $\mathrm{T} 2 \mathrm{w}$, the predicting factor for high-grade lesion.

Shape and margins of lesions seemed to be of a little importance as DCIS usually forms irregular non-mass infiltrates. In our study, only 5 cases out of 114 (4.39\%) formed spiculated lesion and all of them were of high-grade (5 out of $44,11.36 \%)$. There is no statistical significance of this sign in low and high-grade lesion differentiation $(\mathrm{p}=0.14)$, but due

\begin{tabular}{|c|c|c|c|c|}
\hline MR signs & $\begin{array}{l}\text { High-grade } \\
\begin{array}{c}\mathrm{n}=44 \\
(\%)\end{array}\end{array}$ & $\begin{array}{c}\text { Low-grade } \\
\mathrm{n}=33 \\
(\%)\end{array}$ & Odds ratio $(95 \% \mathrm{CI})$ & p-value \\
\hline \multicolumn{5}{|l|}{ T2 Signal } \\
\hline Low & $11(25.0)$ & $2(6.06)$ & $5.17(1.06-25.19)$ & 0.0422 \\
\hline Intermediate & $27(61.36)$ & $14(42.42)$ & $2.16(0.86-5.40)$ & 0.1015 \\
\hline High & $6(13.63)$ & $17(51.52)$ & $0.15(0.05-0.45)$ & 0.0007 \\
\hline \multicolumn{5}{|l|}{ Shape and margins } \\
\hline Irregular & $39(88.64)$ & $33(100)$ & $0.11(0.06$ to 2.01$)$ & 0.1354 \\
\hline Spiculated & $5(11.36)$ & 0 & $9.33(0.50-174.97$ & 0.1354 \\
\hline \multicolumn{5}{|l|}{ Post contrast enhancement } \\
\hline Homogeneous & $2(4.54)$ & $9(27.27)$ & $0.13(0.03-0.64)$ & 0.0121 \\
\hline Non-homogeneous & $42(95.45)$ & $24(72.73)$ & $7.86(1.57-39.48)$ & 0.0121 \\
\hline \multicolumn{5}{|l|}{ „Ring“ enhancement } \\
\hline Yes & 0 & 0 & & \\
\hline No & $44(100)$ & $33(100)$ & $1.33(0.03-68.68)$ & 0.8878 \\
\hline \multicolumn{5}{|l|}{ Ductal enhancement } \\
\hline Yes & $24(54.55)$ & $18(54.55)$ & $1.00(0.40-2.47)$ & 1.0000 \\
\hline No & $20(45.45)$ & $15(45.45)$ & $1.0000(0.4040-2.4756)$ & 1.0000 \\
\hline \multicolumn{5}{|l|}{ Kinetic curves } \\
\hline TIC1 (continuous SI increase) & $8(18.18)$ & $21(64.64)$ & $0.1270(0.0447-0.3607)$ & 0.0001 \\
\hline TIC 2 (plateau) & $19(43.18)$ & $10(30.30)$ & $1.7480(0.6744-4.5306)$ & 0.2504 \\
\hline TIC 3 (“wash-out” phenomenon) & $17(38.63)$ & $2(6.06)$ & $9.7593(2.0644-46.1360)$ & 0.0040 \\
\hline \multicolumn{5}{|l|}{ DWI Signal } \\
\hline Low & $2(4.55)$ & $24(72.73)$ & $0.0179(0.0036-0.0895)$ & $<0.0001$ \\
\hline High & $42(95.45)$ & $9(27.27)$ & $56.00(11.17-280.77)$ & $<0.0001$ \\
\hline \multicolumn{5}{|l|}{ ADC Value } \\
\hline Decrease & $40(90.91)$ & $11(33.33)$ & $20.00(5.69-70.30)$ & $<0.0001$ \\
\hline Increase & $4(9.09)$ & $22(66.67)$ & $0.05(0.01-0.17)$ & $<0.0001$ \\
\hline ADC coefficient $\times 10^{-3} \mathrm{~mm}^{2} / \mathrm{s} \pm \mathrm{SD}$ & $0.87 \pm 0.12$ & $1.09 \pm 0.06$ & $1.00(0.01-92.43)$ & 1.0000 \\
\hline
\end{tabular}


to the high value of Odds ratio $(\mathrm{OR}=9.33)$, spiculated shape might be regarded as a sign supporting high-grade entity.

When analyzing a signal intensity of all 114 DCIS in DWI, the results revealed that presence of restriction of diffusion is a highly reliable parameter in differentiation between high and low-grade. Low signal in DWI appeared in 24 low-grade DCIS out of $33(72.72 \%)$ (Figure 1B), while only 2 highgrade lesions out of $44(4.55 \%, \mathrm{p}<0.0001$, Odds ratio 20.00 , and confidence interval 95\% (CI) 5.69-70.30) demonstrated low signal in DWI. When comparing low and intermediate grade group of DCIS, low signal intensity appeared in 10 cases of intermediate grade group (27.02\%) and in 24 cases of low-grade group $(72.73 \%, \mathrm{p}=0.0002$, Odds ratio 0.14 , CI $95 \%$ 0.048-0.40). High signal in DWI was detected predominantly in intermediate grade group $(72.97 \%$ vs 27.27 , $\mathrm{p}=0.0002$, OR 7.20, CI 95\% 2.06-20.68). In high-grade group, the high signal in DWI was the dominant sign in 42 out of $44(95.45 \%)$, as well as in 27 of intermediate grade lesions (72.97\%, $\mathrm{p}=0.0116$, Odds ratio 7.78 and $95 \%$ CI 1.58-38.27) (Tables 2-4, Figure 3B, Figure 2B).

Figure 1. Low-grade DCIS $(\rightarrow)$ - 58 y old. (A) T2w axial images with fat saturation. (B) DWI axial ep2d; b $=850 \mathrm{~mm}^{2} / \mathrm{s}$. (C) ADC map axial. (D) T1w flash 3D DCE axial with fat saturation. (E) DCE - subtraction image. (F) kinetic curve type 1 , post contrast SI increase of $92.5 \%$.
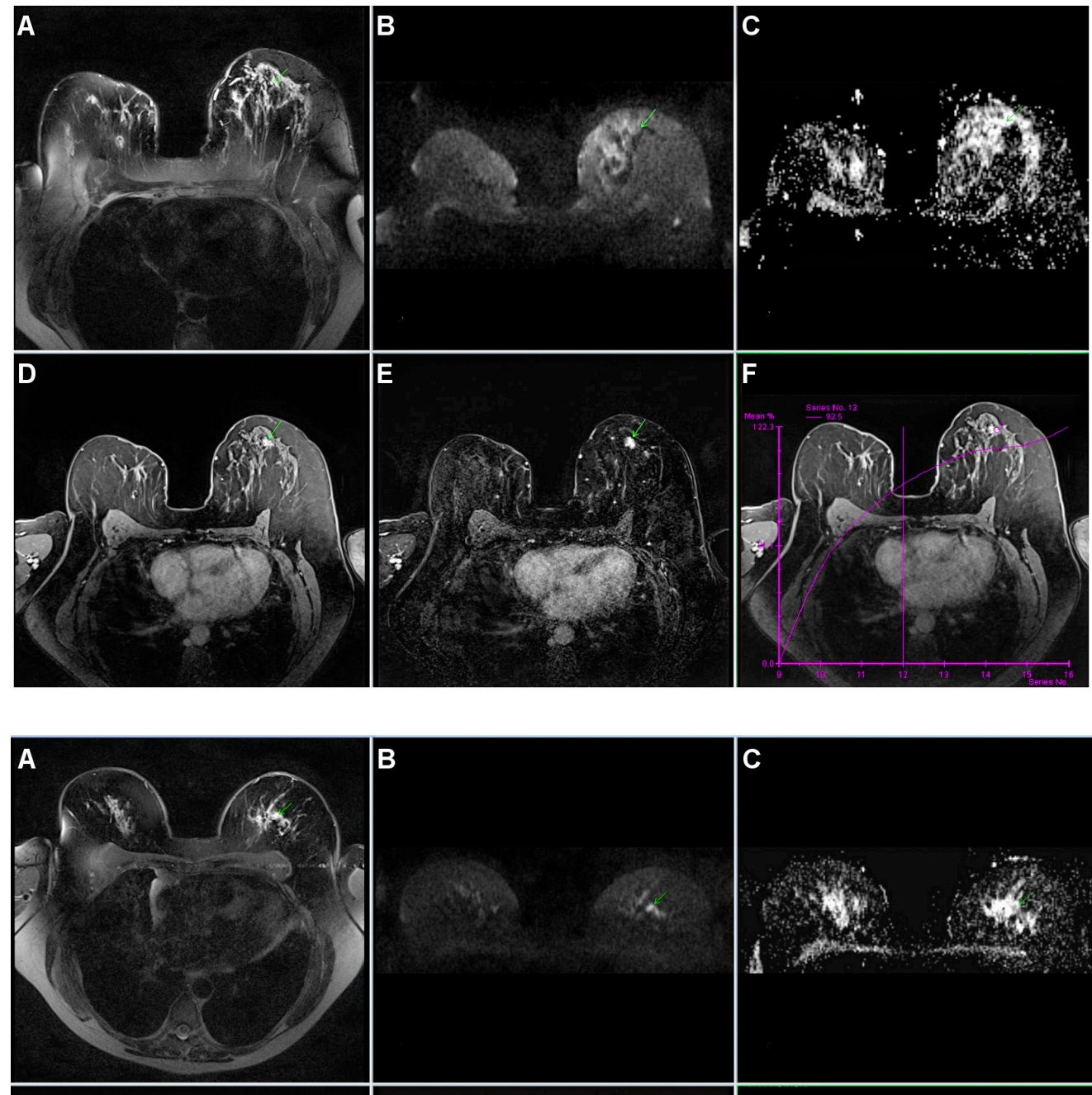

Figure 2. Intermediate DCIS $(\rightarrow)$ - 59 y old. (A) T2w axial images with fat saturation. (B) DWI axial ep2d; b $=850 \mathrm{~mm}^{2} / \mathrm{s}$. (C) ADC map axial. (D) T1w flash 3D DCE axial with fat saturation. (E) DCE - subtraction image. (F) kinetic curve type 2 , post contrast SI increase of $210 \%$.
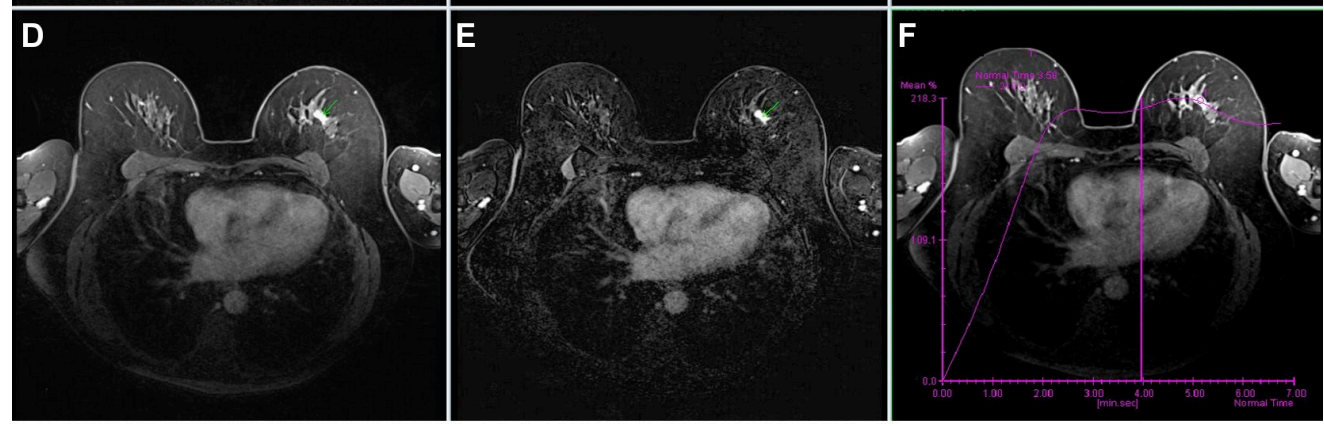

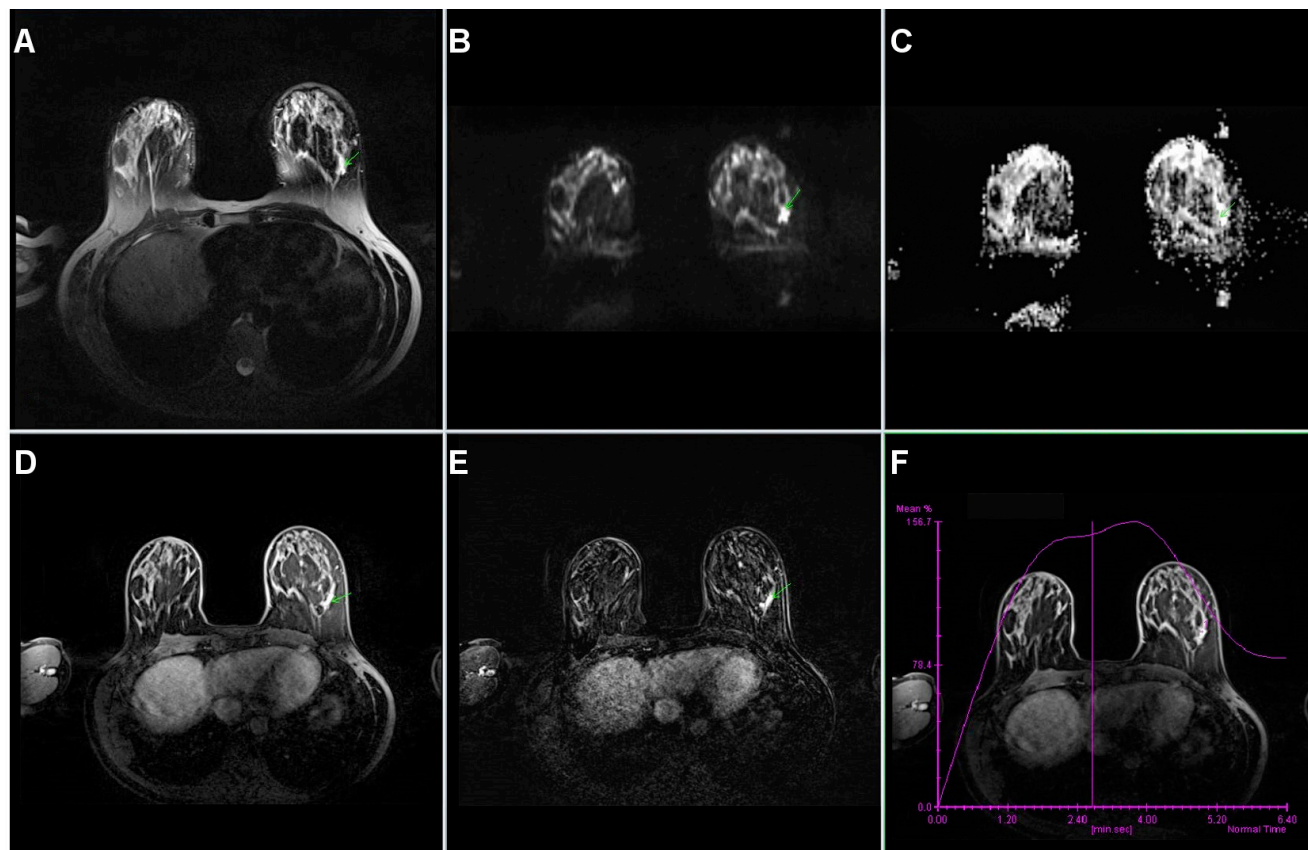

Figure 3. High-grade DCIS $(\rightarrow)$ - 40 y old. (A) T2w axial images with fat saturation. (B) DWI axial ep2d; $b=850 \mathrm{~mm}^{2} / \mathrm{s}$. (C) ADC map axial. (D) T1w flash 3D DCE axial with fat saturation. (E) DCE - subtraction image. (F) kinetic curve type 3 , post contrast SI increase of $149.7 \%$.

Table 3. Results of logistic analysis of morphological and functional MRI signs in correlation with the degree of nuclear atypia for intermediate grade DCIS $(n=37)$ and low-grade DCIS $(n=33)$.

\begin{tabular}{|c|c|c|c|c|}
\hline $\begin{array}{ll}\text { MRI signs } & \text { Degree of nuclear atypia }\end{array}$ & $\begin{array}{l}\text { Intermediate } \\
\quad \mathbf{n}=\mathbf{3 7}\end{array}$ & $\begin{array}{c}\text { Low-grade } \\
\mathbf{n}=33\end{array}$ & $\begin{array}{l}\text { Odds ratio } \\
(95 \% \mathrm{CI})\end{array}$ & p-value \\
\hline \multicolumn{5}{|l|}{ T2 Signal } \\
\hline Low & $7(18.91)$ & $2(6.06)$ & $3.62(0.69-18.83)$ & 0.1267 \\
\hline Intermediate & $17(45.95)$ & $14(42.42)$ & $1.03(0.40-2.69)$ & 0.9484 \\
\hline High & $13(35.14)$ & $17(51.52)$ & $0.51(0.20-1.33)$ & 0.1690 \\
\hline \multicolumn{5}{|l|}{ Shape and margins } \\
\hline Regular & 0 & 0 & & \\
\hline Lobular & 0 & 0 & & \\
\hline Spiculated & 0 & 0 & & \\
\hline Irregular & $37(100)$ & $33(100)$ & $1.12(0.02-58.00)$ & 0.9553 \\
\hline \multicolumn{5}{|l|}{ Post contrast enhancement } \\
\hline Homogeneous & $7(18.91)$ & $9(27.27)$ & $0.62(0.20-1.92)$ & 0.4081 \\
\hline Non-homogeneous & $30(81.08)$ & $24(72.73)$ & $1.61(0.52-4.95)$ & 0.4081 \\
\hline \multicolumn{5}{|l|}{ „Ring“ enhancement } \\
\hline yes & 0 & 0 & & \\
\hline no & $37(100)$ & $33(100)$ & $1.12(0.02-58.00)$ & 0.9553 \\
\hline \multicolumn{5}{|l|}{ Ductal enhancement } \\
\hline yes & $21(56.76)$ & $18(54.55)$ & $1.4(0.43-2.81)$ & 0.8525 \\
\hline no & $16(43.24)$ & $15(45.45)$ & $0.91(0.36-2.35)$ & 0.8525 \\
\hline \multicolumn{5}{|l|}{ Kinetic curves } \\
\hline TIC 1 (continuous increase) & $9(24.32)$ & $21(63.64)$ & $0.18(0.07-0.52)$ & 0.0013 \\
\hline TIC 2 (plateau) & $21(56.76)$ & $10(30.30)$ & $3.02(1.13-8.10)$ & 0.0282 \\
\hline TIC3 (“wash-out" phenomenon) & $7(18.92)$ & $2(6.06)$ & $3.62(0.69-18.83)$ & 0.1267 \\
\hline \multicolumn{5}{|l|}{ DWI Signal } \\
\hline Low & $10(27.02)$ & $24(72.73)$ & $0.14(0.05-0.40)$ & 0.0002 \\
\hline High & $27(72.97)$ & $9(27.27)$ & $7.20(2.51-20.68)$ & 0.0002 \\
\hline \multicolumn{5}{|l|}{ ADC Value } \\
\hline Decrease & $25(67.57)$ & $11(33.33)$ & $4.17(1.53-11.31)$ & 0.0051 \\
\hline Increase & $12(32.43)$ & $22(66.67)$ & $0.24(0.09-0.65)$ & 0.0051 \\
\hline ADC coefficient $\times 10^{-3} \mathrm{~mm}^{2} / \mathrm{s} \pm \mathrm{SD}$ & $0.95 \pm 0.10$ & $1.09 \pm 0.06$ & $0.33(0.07-16.80)$ & 0.5828 \\
\hline
\end{tabular}


Table 4. Results of the logistic regress analysis of morphological and functional MRI signs in correlation with the degree of nuclear atypia for high-grade DCIS $(n=44)$ and intermediate grade DCIS $(n=37)$.

\begin{tabular}{|c|c|c|c|c|}
\hline MRI signs & $\begin{array}{c}\text { High-grade } \\
\mathrm{n}=44 \\
(\%)\end{array}$ & $\begin{array}{c}\text { Intermediate } \\
\mathbf{n}=37 \\
(\%)\end{array}$ & $\begin{array}{c}\text { Odds ratio } \\
(95 \% \mathrm{CI})\end{array}$ & p-value \\
\hline \multicolumn{5}{|l|}{ T2 Signal } \\
\hline Low & $11(25)$ & $7(18.91)$ & $1.43(0.49-4.16)$ & 0.5131 \\
\hline Intermediate & $27(63.36)$ & $17(45.95)$ & $1.87(0.77-4.54)$ & 0.1670 \\
\hline High & $6(13.64)$ & $13(35.14)$ & $0.29(0.10-0.87)$ & 0.0272 \\
\hline \multicolumn{5}{|l|}{ Shape and margins } \\
\hline Regular & 0 & 0 & & \\
\hline Lobular & 0 & 0 & & \\
\hline Irregular & $39(100)$ & $37(100)$ & $0.10(0.06-1.79)$ & 0.1165 \\
\hline Spiculated & $5(11.36)$ & 0 & $10.44(0.56-195.46)$ & 0.1165 \\
\hline \multicolumn{5}{|l|}{ Post contrast enhancement } \\
\hline Homogeneous & $2(4.55)$ & $7(18.92)$ & $0.20(0.04-1.05)$ & 0.0575 \\
\hline Non-homogeneous & $42(95.45)$ & $30(82.08)$ & $4.90(0.95-25.26)$ & 0.0575 \\
\hline \multicolumn{5}{|l|}{ „Ring“ enhancement } \\
\hline yes & 0 & 0 & & \\
\hline no & $44(100)$ & $37(100)$ & $1.19(0.02-61.26)$ & 0.9322 \\
\hline \multicolumn{5}{|l|}{ Ductal enhancement } \\
\hline yes & $24(54.55)$ & $21(56.76)$ & $0.91(0.38-2.21)$ & 0.8419 \\
\hline no & $20(45.45)$ & $16(43.24)$ & $1.09(0.45-2.64)$ & 0.8419 \\
\hline \multicolumn{5}{|l|}{ Kinetic curves } \\
\hline TIC 1 (continuous increase) & $8(18.18)$ & $9(24.32)$ & $0.69(0.24-2.02)$ & 0.5001 \\
\hline TIC 2 (plateau) & $19(43.18)$ & $21(56.76)$ & $0.58(0.24-1.40)$ & 0.2250 \\
\hline TIC 3 ("wash-out" phenomenon) & $17(38.64)$ & $7(18.92)$ & $2.70(0.97-7.50)$ & 0.0570 \\
\hline \multicolumn{5}{|l|}{ DWI Signal } \\
\hline Low & $2(2.54)$ & $10(54.05)$ & $0.13(0.03-0.63)$ & 0.0116 \\
\hline High & $42(95.45)$ & $27(72.97)$ & $7.78(1.58-38.27)$ & 0.0116 \\
\hline \multicolumn{5}{|l|}{ ADC Value } \\
\hline Decrease & $40(90.91)$ & $25(67.56)$ & $4.80(1.39-16.54)$ & 0.0129 \\
\hline Increase & $4(9.09)$ & $12(32.44)$ & $0.21(0.06-0.72)$ & 0.0129 \\
\hline ADC coefficient $\times 10^{-3} \mathrm{~mm}^{2} / \mathrm{s} \pm \mathrm{SD}$ & $0.87 \pm 0.12$ & $0.95 \pm 0.10$ & $0.33(0.00-52.57)$ & 0.6705 \\
\hline
\end{tabular}

These results correlate with further observations in ADC map. Low signal intensity as an indicator of restriction of diffusion appeared in 40 cases of high-grade $(90.91 \%$, $\mathrm{p}<0.0001$ ) (Figure 3C), while only in 11 cases of low-grade DCIS (33.33\%). In low-grade group, the absence of restriction of diffusion (high signal in ADC map) was observed in 22 out of 33 (66.67\%) (Figure 1C), while only in 4 cases of high-grade lesions $(9.09 \%, \mathrm{p}<0.0001$, OR 56.00 , CI $95 \%$ $11.17-280.77)$ and in 12 out of 37 cases of intermediate DCIS (32.43\%, p $<0.0051$, OR 0.24, CI 95\% 0.09-0.65) (Figure 2C). Value of ADC coefficient (at $\mathrm{b}=0,50,850$ ) didn't play any significant role in differentiation between any groups of DCIS.

The analysis of post contrast dynamic study of all DCIS revealed very heterogeneous results. Only 26 lesions (22.81\%) had kinetic curve TIC type III with wash out phenomenon. There was a significant difference between the high and low-grade lesions - in case of high-grade, 17 out of $44(38.64 \%)$ reported kinetic curve type III with wash-out (Figure 3D-F), in the group of low-grade DCIS it appeared only in 2 out of $33(6 \%, p=0.004$, OR 9.76, CI $95 \%$ 2.06-46.14). When comparing groups of intermediate and low-grade DCIS in post contrast dynamic study, another statistically significant sign was recognized - the kinetic curve type I supported the diagnosis of low-grade lesion (64.63\% vs $24.32 \%, \mathrm{p}=0.0013$, OR 0.18 , CI $95 \% 0.065-0.52$ ) (Figure 1D-F) and type II the diagnosis of the intermediate grade DCIS ( $56.76 \%$ vs $30.30 \%$, $\mathrm{p}=0.0282,56.76 \%$, OR 3.02 , CI 95\% 1.13-8.10) (Figure 2D-F). Kinetic curve type III as a sign for differentiation between low and intermediate lesions didn't reach the statistical significance (low-grade 6.06\% vs intermediate grade $18.91 \%, \mathrm{p}=0.1267$, OR 3.62 , CI $95 \%$ 0.69-18.83).

Ductal enhancement which appeared identically in both high and low-grade groups in $54.55 \%(\mathrm{p}=1.000$, OR 1.00 , CI 95\% 0.40-2.48) was found to be statistically non-significant. Homogeneity of post contrast enhancement revealed the most significant differences only in distinction of high 
and low-grade DCIS, where high-grade DCIS lesions were enhancing non-homogeneously in 42 out of 44 cases (95.45\%) and only in 2 cases (4.54\%) homogeneously. Low-grade DCIS enhanced dominantly non-homogeneously (24 cases, $72.72 \%$ ) but in 9 cases (27.27\%) homogeneous enhancement appeared $(\mathrm{p}=0.0121)$. When comparing other groups, the homogeneity or non-homogeneity of enhancement appeared there comparatively and so this sign couldn't be regarded as a marker of degree of nuclear atypia.

\section{Discussion}

Ductal carcinoma in situ comprises heterogeneous lesions, which can be according to the degree of nuclear atypia divided to low, intermediate and high-grade by histopathological analysis. In MRI their characteristics overlap. The combination of morphological, functional and molecular information offered by multiparametric MRI promises to make it a successful tool for improving many aspects of breast cancer diagnosis [9]. The unique role of MRI in the diagnostics of DCIS is still discussed and its benefit for further management is examined carefully by studies (finished studies regarding this question - COMICE, MONET, currently running study MIPA) [10-12]. However, MRI features seem to be important for the proper management of DCIS, especially when concerning its potential to progress into invasive tumor [13].

Detailed analysis revealed that the most prominent differences of characteristics are between the low-grade and highgrade lesions. This is in a concordance with the observations of pathologists: the diagnostic criteria for low and high-grade lesions are strictly defined. Intermediate grade lesions stay between these two groups and their characteristics don't belong to any of these groups and the differences are very subtle [14].

The most reliable signs for differentiation between high and low-grade group according to the calculation of statistical significance $(p)$ and Odds ratio were signal intensity in DWI and in ADC map together with post contrast behavior of lesions in dynamic analysis.

High signal intensity of DCIS in DWI together with the presence of low signal in ADC is reliable parameter supporting the high-grade diagnosis. DWI works on the principle of measurement of water molecular diffusion between different environments and indirectly determines the degree of lesion cellularity [15]. The same results about the role of DWI in detections of DCIS was also revealed in the study of Rahbar et al. [16]. Authors proved that the higher signal intensity in DWI is a sign supporting DCIS, even though they didn't used their results for further differentiation between the degrees of nuclear atypia. Other study [17, 18] was aimed at differentiation between DCIS and invasive cancer. It proved the potential of DWI to predict invasion by increased SI and this indirectly supports the theory that higher grade with higher potential to invasive spreading is characterized by higher SI at DWI.
Character of enhancement of DCIS in post contrast dynamic study is very variable and when analyzing 114 cases of DCIS, all three types of kinetic curves were identified. Interesting is the fact that the kinetic curve type III with a "wash out phenomenon", which is regarded to be the most reliable indicator of malignancy [19], appeared only in one fifth of all cases. "Wash out phenomenon" was dominantly observed in high-grade group, followed by intermediate grade. Type I appeared exclusively only in low-grade lesions. The same results appeared in the studies of other authors $[1,20-22]$. On the other side, continuous increase of signal intensity with kinetic curve type I is according to our results the sign supporting low-grade of nuclear atypia. The observation could be explained according to some authors by the fact that during the gradual transformation of benign lesion to DCIS and finally to invasive carcinoma, the increase of perfusion in the tumor as a result of increased vascular density can be observed $[23,24]$. The difference of enhancement between the groups of DCIS results from the impaired permeability of basal membrane [19], which seems to be more prominent in high-grade lesions.

Other examined post contrast parameters - ductal and ring enhancement - didn't reach the statistical significance and thus cannot be regarded as a reliable sign for determination of degree of atypia. The ring enhancement wasn't detected in any case of DCIS. This is in agreement with the knowledge that DCIS is usually presented by non-mass enhancement $[25,26]$, while ring enhancement is a pattern typical for focal and frequently invasive lesion [27]. The presence of ductal enhancement was distributed comparatively in all three groups and we observed that the sensitivity of MR mammography in DCIS detection correlates with the degree of nuclear atypia. This means it grows with the probability of the transformation to invasive form [28] due to the increased enhancement. On the other side, the fact that low-grade lesions may stay unenhanced and thus undetectable often makes the diagnostics of this entity challenging or even more problematic.

Irregular, spiculated shape is not a typical sign of DCIS and usually appears in case of invasive carcinoma. This fact correlates also with our outcomes considering that only 5 high-grade lesions out of all 114 formed spiculated lump. According to some authors spiculated shape might predict the very pre-invasive phase of high-grade DCIS [29].

According to the results of our retrospective multiparametric study, we can proclaim that the extended MR protocol (using DWI) has the potential to predict the degree of nuclear atypia, especially in differentiation between high and low-grade DCIS. The most reliable signs of high-grade DCIS are low T2 signal, high signal intensity in DWI, presence of restriction of diffusion in ADC map, non-homogeneous post contrast enhancement and kinetic curve type III. These parameters support the presence of high degree nuclear atypia and thus can predict the higher risk of transformation of DCIS into invasive carcinoma. 


\section{References}

[1] KUHL CK. Why do purely intraductal cancers enhance on breast MR images? Radiology 2009; 253: 281-283. https:// doi.org/10.1148/radiol.2532091401

[2] RECHT A, RUTGERS EJ TH, FENTIMAN IS, KURTZ JM, MANSEL E et al. The fourth EORTC DCIS Consensus Meeting (Heemskerk, The Netherlands, 23-24 January 1998); conference report. Eur J Cancer 1998; 34: 1664-1669.

[3] MOSSA-BASHA M, FUNDARO GM, SHAH B A, ALI S, PANTELIC MV. Ductal carcinoma in situ of the breast: MR imaging findings with histopathologic correlation. Radiographics 2010; 30: 1673-1687. https://doi.org/10.1148/ rg.306105510

[4] KUHL CK. Current Status of Breast Imaging. Part 2. Clinical applications. Radiology 2007; 244: 672-691. https://doi. org/10.1148/radiol.2443051661

[5] JANSEN SA, NEWSTEAD GM, ABE H, SHIMAUCHI A, SCHMIDT RA et al. Pure ductal carcinoma in situ: kinetic and morphologic MR characteristics compared with mammographic appearance and nuclear grade. Radiology 2007; 245: 684-691. https://doi.org/10.1148/radiol.2453062061

[6] HOUSSAMI N, CIATTO S, MACASKILL P, LORD SJ, WARREN RM et al. Accuracy and surgical impact of magnetic resonance imaging in breast cancer staging: systematic review and meta-analysis in detection of multifocal and multicentric cancer. J Clin Oncol 2008; 26: 3248-3258. https:// doi.org/10.1200/JCO.2007.15.2108

[7] LEHOTSKA V. [MRI in the diagnosis of breast lesions]. In: J. Bilicky, J. Slobodnikova, V. Lehotska (Eds), Radiologia III. Veda SAV Bratislava, 2009, pp 147. ISBN 9788022410670.

[8] LEHOTSKA V. [Value and feasibility of magnetic resonance imaging (MR-mammography) in the diagnosis of breast lesions]. Onkologia 2007; 2: 211-214.

[9] PINKER K, BICKEL H, HELBICH THE, GRUBER S, DUBSKY P et al. Combined contrast-enhanced magnetic resonance and diffusion-weighted imaging reading adapted to the "Breast Imaging Reporting and Data System" for multiparametric 3-T imaging of breast lesions. Eur Radiol 2013; 23: 1791-1802. https://doi.org/10.1007/s00330-013-2771-8

[10] TURNBULL L, BROWN S, HARVEY I, OLIVIER C, DREW $P$ et al. Comparative effectiveness of MRI in breast cancer (COMICE) trial: a randomised controlled trial. Lancet 2010; 375: 563-571. https://doi.org/10.1016/S01406736(09)62070-5

[11] PETERS NH, VAN ESSER S, VAN DEN BOSCH MA, STORM RK, PLAISIER PW et al. Preoperative MRI and surgical management in patients with nonpalpable breast cancer: the MONET - randomised controlled trial. Eur J Cancer 2011; 47: 879-886. https://doi.org/10.1016/j. ejca.2010.11.035

[12] TRIMBOLI RM, DI LEO G, SACCHETTO D, MANN R, ALVAREZ BENITO $\mathrm{M}$ et al. The impact of breast MRI on surgical planning and reoperation rate: first results from the MIPA study. Insights Imaging 2016; 7 (Suppl 1): S464. https://doi.org/10.1007/s13244-016-0475-8
[13] BAUER A, BAHRS SD, SPECK S, WIETEK BM, KRAMER $B$ et al. Breast MRI of pure ductal carcinoma in situ: sensitivity of diagnosis and influence of lesion characteristics. Eur J Radiol 2013; 82: 1731-1737. https://doi.org/10.1016/j. ejrad.2013.05.002

[14] BANE A. Ductal Carcinoma In Situ: What the Pathologist Needs to Know and Why. Int J Breast Cancer 2013; 2013: 914053. https://doi.org/10.1155/2013/914053

[15] YOSHIKAWA MI, OHSUMI S, SUGATA S, KATAOKA M, TAKASHIMA $S$ et al. Relation between cancer cellularity and apparent diffusion coefficient values using diffusion-weighted magneticresonanceimaginginbreastcancer. RadiatMed 2008; 26: 222-226. https://doi.org/10.1007/s11604-007-0218-3

[16] RAHBAR H, PARTRODGE SC, EBY PR, DEMARTINI WB, GUTIERREZ RL et al. Characterization of ductal carcinoma in situ on diffusion weighted breast MRI. Eur Radiol 2011; 21: 2011-2019. https://doi.org/10.1007/s00330-011-2140-4

[17] BICKEL H, PINKER-DOMENIG K, BOGNER W, SPICK C, BAGO-HORVATH ZS et al. Quantitative apparent diffusion coefficient as a noninvasive imaging biomarker for the differentiation of invasive breast cancer and ductal carcinoma in situ. Invest Radiol 2015; 50: 95-100. https://doi.org/10.1097/ RLI.0000000000000104

[18] CHOI YS, CHANG YW, PARK HJ, KIM HJ, HONG SS et al. Correlation of the apparent diffusion coefficient values on diffusion-weighted imaging with prognostic factors for breast cancer. Br J Radiol 2012; 85: e474-479. https://doi. org/10.1259/bjr/79381464

[19] OREL SG: Differentiating benign from malignant enhancing lesions identified at MR imaging of the breast: are time-signal intensity curves an accurate predictor? Radiology 1999; 211: 5-7. https://doi.org/10.1148/radiology.211.1.r99ap395

[20] NEUBAUER H, LIM, KUEHNE-HEID R, SCHNEIDER A, KAISER WA. High-grade and non-high-grade ductal carcinoma in situ on dynamic MR-mammography: Characteristic findings for signal increase and morphological pattern of enhancement. Br J Radiol 2003; 76: 3-12. https://doi. org/10.1259/bjr/14883856

[21] VAN GOETHEM M, SCHELFHOUT K, KERSCHOF E, COLPAERT C, WEYLER J et al. Comparison of MRI features of different grades of DCIS and invasive carcinoma of the breast. JBR-BTR 2005; 88: 225-232.

[22] JANSEN SA, PAUNESKU T, FAN X, WOLOSCHAK GE, VOGT $S$ et al. Ductal carcinoma in situ: $\mathrm{x}$-ray fluorescence microscopy and dynamic contrast-enhanced MR imaging reveals gadolinium uptake within neoplastic mammary ducts in a murine model. Radiology 2009; 253: 399-406. https://doi.org/10.1148/radiol.2533082026

[23] FURMAB-HARAN E, SCHECHTMAN E, KELCZ F, KIRSHENBAUM K, DEGANI H: Magnetic Resonance Imaging reveals functional diversity of the vasculature in benign and malignant breast lesions. Cancer 2005; 104: 708-718. https:// doi.org/10.1002/cncr.21225

[24] FROUGE C, GUINEBRETIERE JM, CONTESSO G, DI PAOLA R, BLERY M: Correlation between contrast enhancement in dynamic magnetic resonance imaging of the breast and tumor angiogenesis. Invest Radiol 1994; 29: 1043-1049. 
[25] MACURA KJ, OUWERKERK R, JACOBS MA, BLUEMKE DA. Patterns of enhancement on Breast MR Images: Interpretation and Imaging Pitfalls. Radiographics 2006; 26: 1719-1734. https://doi.org/10.1148/rg.266065025

[26] NEWELL D, NIE K, CHEN JH, HSU CC, YU HJ et al. Selection of diagnostic features on breast MRI to differentiate between malignant and benign lesions using computer-aided diagnosis: differences in lesions presenting as mass and non-mass-like enhancement. Eur Radiol 2010; 20: 771-781. https://doi.org/10.1007/s00330-009-1616-y
[27] HELBICH TH: Contrast-enhanced magnetic resonance imaging of the breast. Eur J Radiol 2000; 34: 208-219.

[28] KUHL CK, SCHRADING S, BIELING HB, WARDELMANN E, LEUTNER CC et al. MRI for diagnosis of pure ductal carcinoma in situ: a prospective observational study. Lancet 2007; 370: 485-492. https://doi.org/10.1016/S01406736(07)61232-X

[29] BADRUDDOJA M: Ductal Carcinoma of the Breast. A Surgical Perspective. Int J Surg Oncol 2012; 2012: 761364. https://doi.org/10.1155/2012/761364 